\title{
Kinematic studies of the IDV quasar 0917+624
}

\author{
Simone Bernhart* \\ Max-Planck-Institut für Radioastronomie, Bonn, Germany \\ E-mail: simoneempifr-bonn.mpg.de \\ Thomas P. Krichbaum \\ Max-Planck-Institut für Radioastronomie, Bonn, Germany \\ E-mail: tkrichbaume@mpifr-bonn.mpg.de

\section{Lars Fuhrmann} \\ Max-Planck-Institut für Radioastronomie, Bonn, Germany \\ E-mail: Ifuhrmann@mpifr-bonn.mpg.de
}

\section{Alexander Kraus}

Max-Planck-Institut für Radioastronomie, Bonn, Germany

E-mail: akraus@mpifr-bonn.mpg.de

For more than a decade from the late 1980s to the late 1990s, the intraday variable (IDV) quasar $0917+624$ was known to be strongly variable with amplitude variations of $10-20 \%$ within 0.8 to 1.6 days. During that period it showed faster variability in the polarized flux and the polarization angle than in total intensity. However, since 2000 the IDV of this source has almost ceased, attaining a variability index of only less than a few percent. If interstellar scintillation was responsible for the IDV in the past, one possible explanation for the sudden change of the variability mode could be an intrinsic change in the morphology of the source, such as an increase in the size of the structural components that are responsible for the scintillation. New jet components appearing at the jet base could lead to a temporarily larger size of the scintillating component when compared to the scattering size of the interstellar medium, causing quenched scintillation. In order to test this scenario, VLBI observations of 0917+624 from 1999 to 2005 have been analysed. Here, we summarize first results and discuss them in the framework of the quenched scintillation model.

The 8th European VLBI Network Symposium

September 26-29, 2006

Toruń, Poland

\footnotetext{
* Speaker.
} 


\section{Introduction}

Extragalactic compact flat-spectrum radio sources are known to be highly variable and about $30 \%$ of them show intra-day variability (IDV) (e.g., [1],[2],[3]). 0917+624 ( $\mathrm{z}=1.446)$ is a typeII IDV quasar. (See [ $[$ t for a more detailed description of IDV). The source used to be strongly variable on timescales of 0.8 to 1.6 days with modulation indices $m^{1}$ of 3 to $5 \%$ at cm-wavelengths from 1986 to 1998. Moreover, in the polarized flux, as well as in the polarization angle, even faster variability was detected ([5]); the polarized flux usually being anti-correlated with the total flux ([6). The source structure on milliarcsecond scales reveals a compact core with a jet pointing in northerly direction (see right panel of Figure 1), whereas on arcsecond scales the source is mainly pointlike showing a jet-like feature 4 mas to the south-west (e.g., [7]).

In September 1998, Kraus et al. ([8]) discovered that the rapid variability had stopped and instead a slow, monotonic increase of $7 \%$ in total flux density was observed during the 5-day observing session. Observations made in February 1999 revealed that $0917+624$ was varying again on a time scale of 1.3 days. However, since September 2000 the variability of the source has ceased again, showing only moderate modulation indices of the order of $\sim 0.5 \%$ (see [9], [10]), and to date the source has not restarted its former activity.

The question whether the origin of IDV is source extrinsic, i.e. due to scintillation in the interstellar medium (ISM), or intrinsic, remains unanswered. It is expected, though, that IDV blazar cores feature micro- or even nanoarcsecond angular sizes and therefore must scintillate through the ISM. Whether it be extrinsic or intrinsic, possible explanations for the reduced IDV activity could be either that the scintillating medium has changed (e.g., strength of turbulence, distance), or the flux of the scintillating component(s) has now decreased and become less dominant. Another possibility is the precession of the footpoint of the jet, which has been proposed in the cases of $0716+714$ [11] and 1803+784 [12], i.e. the size of the VLBI core changes with time due to precession.

Furthermore, the earlier slow-down of 0917+624 in September 1998 was interpreted by Kraus et al. ([8]) as probably due to either a disappearance of the scintillating compact component or an increase in its angular size, which could be caused by the ejection of a new jet component (see also [13]). The ejection could temporarily lead to a core size exceeding the Fresnel scale defined by the scattering medium. Consequently, only strongly quenched scintillation is observed. When the component moves further down the jet, it will separate from the core at some point whereby the size of the scintillating component decreases again. At that time, the variability is expected to reappear. Given that the variability ceased again in 2000, even more than one component should have been emitted since. In order to test this scenario, new VLBI observations of 0917+624 were carried out at $6 \mathrm{~cm}, 2 \mathrm{~cm}$ and $1.3 \mathrm{~cm}$ at 4 epochs, covering the period between December 2001 and April 2003. Here, we present the results of the analysis of our 2-cm data complemented with additional 2-cm data, including 3 VLBI epochs observed between November 1999 and November 2000 ([9], [13]) and 6 epochs from the MOJAVE ${ }^{2}$ ([14]) and 2cm Survey ([15]) programs observed between June 2002 and August 2006. A more detailed description of the observations and the data reduction will be given in Fuhrmann et al. in prep.

\footnotetext{
${ }^{1} m[\%]=\sigma_{S} /<S>$, where $\sigma_{S}$ is the rms flux density

${ }^{2}$ Monitoring of Jets in Active galactic nuclei with VLBA Experiments
} 

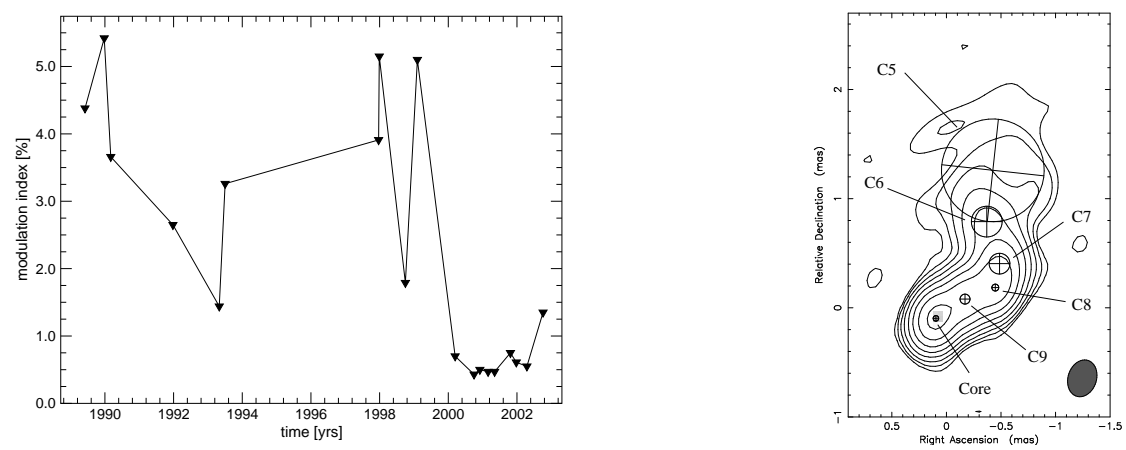

Figure 1: Left: Modulation index of 0917+624 versus time from 1989 to 2002; Right: VLBI map of $0917+624$ at $2 \mathrm{~cm}$ observed in April 2003, beam $0.345 \times 0.261$ mas, beam position angle $-18^{\circ}$; the map is a representative example for the component identification in all epochs.

\section{Results and discussion}

\subsection{Kinematic analysis}

To investigate the jet kinematics of $0917+624$ we fitted a number of circular Gaussian components to the calibrated visibilities in order to describe the source with as few parameters as possible. For each epoch we cross-identified individual model components along the jet using their distance from the VLBI core, which is assumed to be stationary, the flux density and size. The right panel of Figure 1 shows a representative map of the component identification. The higher numbered components are the ones that were expelled last. By means of a graphical analysis, which is presented in the left panel of Figure 2, we obtained an adequate identification scheme for the kinematics in the jet of $0917+624$. This scenario consists of 10 superluminal components in total, moving linearly away from the core, the innermost 7 of which are displayed here.

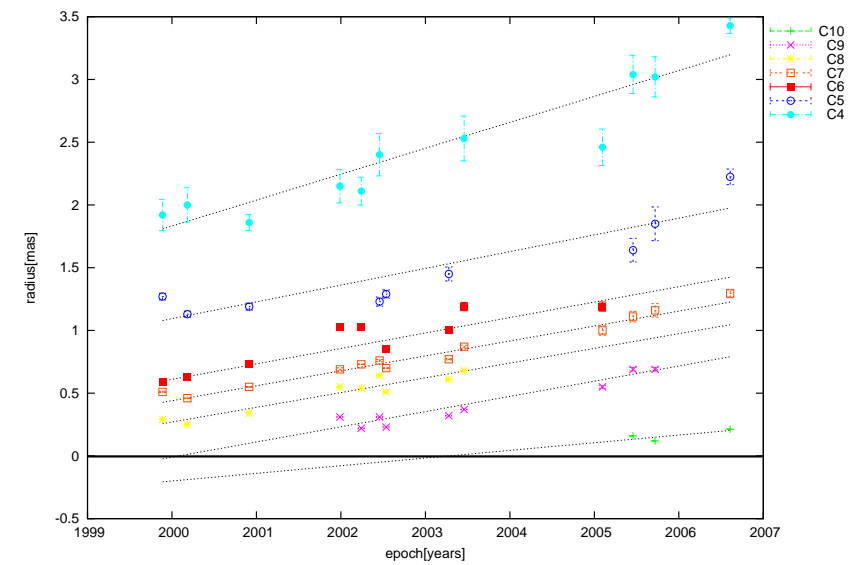

\begin{tabular}{c|c} 
Component & $\beta_{a p p} \pm \Delta \beta_{a p p}[\mathrm{c}]$ \\
\hline C4 & $12.97 \pm 1.54$ \\
C5 & $8.38 \pm 1.89$ \\
C6 & $7.74 \pm 0.97$ \\
C7 & $7.47 \pm 0.48$ \\
C8 & $7.37 \pm 0.62$ \\
C9 & $7.61 \pm 2.00$ \\
C10 & $3.83 \pm 2.61$
\end{tabular}

Figure 2: Core separation of the individual modelfit components plotted versus time; the solid lines are linear fits to the path of each component; from the slope the apparent velocities are derived which are listed in the Table.

From the slope of the linear fit to the path of the components, the individual apparent velocities can be calculated. The results are listed in the Table of Figure 2. The inner components C9, C8, C7, C6, C5 move with an apparent speed of $\beta_{a p p}=7.4$ to 8.4 (adopting $H_{0}=73 \mathrm{kms}^{-1} \mathrm{Mpc}^{-1}$ ), which is 


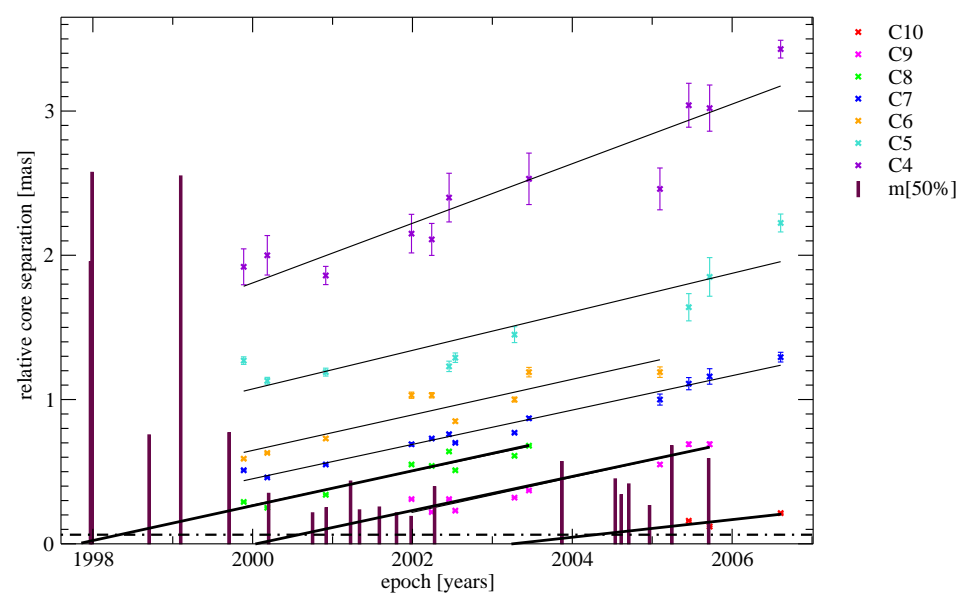

Figure 3: Core separation plotted with modulation indices (scaled down by 50\%) displayed as bars; the dashed-dotted line indicates the typical scattering size $(\sim 0.07$ mas) for this source as defined by the ISM (see [16])

in good agreement with previous findings ([13]). Moreover, a back-extrapolation of the fitted line to the time-axis delivers an approximate ejection date for the respective component (see Figure 3). For component C8, the ejection date lies around 1997.8, which is in the range of earlier results found by Krichbaum et al. [13] and Fuhrmann [9]. That event corresponds to a high level of variability. The modulation index lies at $\sim 5 \%$ and decreases in the aftermath to $\sim 1.5 \%$. That was about the time when the size of the core and the emitted component exceed the typical scattering size of the medium (indicated by the dashed-dotted line in Figure 3 ), presumably resulting in strongly quenched scintillation. Considering the succeeding increase in the modulation index in early 1999, the behaviour of $\mathrm{C} 8$ could in principle confirm our working hypothesis.

Component C9 was approximately ejected in the beginning of 2000, which overlaps with a period of very low variability according to the modulation indices around that time. The data for the last but two observing epoch reveal that another component (C10), which was not visible in the earlier epochs, has been emitted moving with an apparent speed of $\beta_{a p p}=3.83 \pm 2.61$. The backextrapolation yields an approximate ejection date of 2002.3. However, ejection date and apparent speed are affected by a high uncertainty due to a large $(>50 \%)$ error in the slope of the component path, since only three-epoch measurements are available for $\mathrm{C} 10$ so far.

\subsection{Flux density measurements}

Theoretical simulations have shown that the ejection of a new VLBI component can be accompanied by a flux outburst at all frequencies [17]. The flux reaches maximum when the component becomes optically thin, which depends on the opacity at each frequency. The flux peak is higher and emerges earlier at higher frequencies. This general behaviour in flux outburst at various frequencies is shown in Figure 4 where long-term lightcurves of 0917+624 at 7 different frequencies (from 1.3 to $18 \mathrm{~cm}$ ) are displayed. The data were obtained from observations with the Effelsberg $100-\mathrm{m}$ radio telescope and also complemented by $\mathrm{UMRAO}^{3}$ data. From the slope after a major

\footnotetext{
${ }^{3}$ University of Michigan Radio Astronomy Observatory
} 


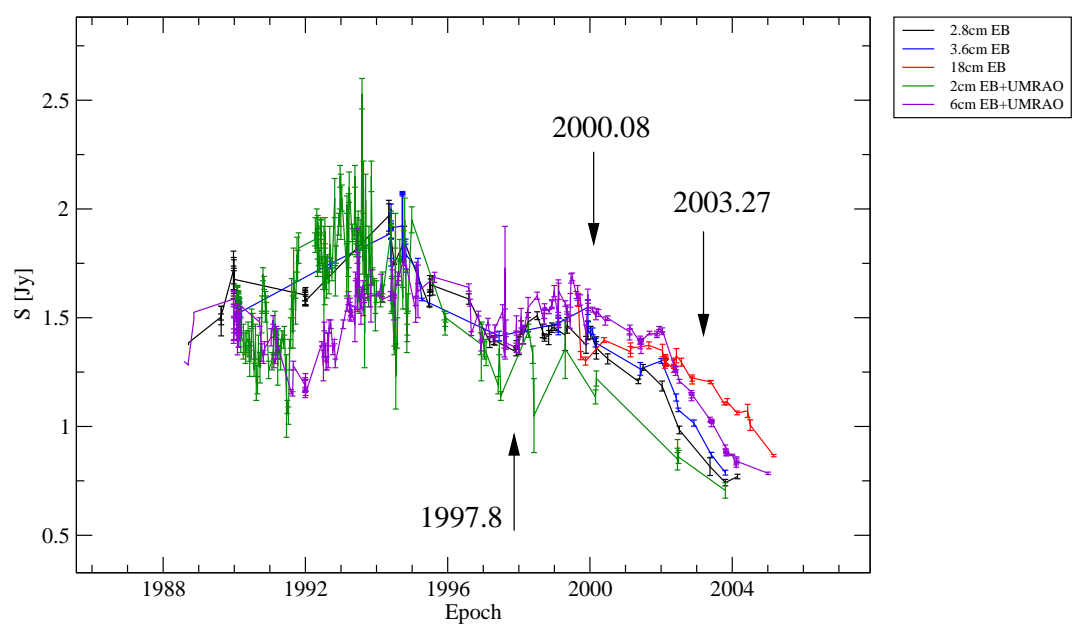

Figure 4: Multi-frequency plot of flux density observations carried out with the Effelsberg radio telescope and extracted from the UMRAO data base; the arrows indicate the ejection dates of new jet components.

flare, the time delay between the highest and lowest frequency can be derived. This gives an approximation of the period that a newly ejected component needs to separate from the VLBI core. After this time, the IDV activity is expected to resume. In the case of $0917+624$, the delay is approximately 0.8 years (N. Kudryavtseva, priv. comm.) for the large flare around 2000, which coincides with the ejection date we found for component C9. This implies that at least one year after this flare, the source should exhibit a higher level of IDV activity again, which is, however, not the case according to the modulation indices around 2001 (see Figure $\bigoplus$ ). Furthermore, it seems that a burst in flux that is related to a new component ejection, is not directly related to the IDV activity of a source, since the modulation index was comparatively low at the time of the flare.

\section{Conclusion}

We found two new VLBI components being ejected around the beginning of 2000 and possibly in early 2003. The gap between the two ejection dates is too long compared to the time obviously needed for a component to travel down the jet and separate from the core. Therefore, the IDV activity should meanwhile have resumed. Since this is not the case, the hypothesis of a quenched scintillation scenario is not conclusive. Taking the existing data, we will now focus on the alternative scenarios, e.g., the analysis of changes in the flux density of the scintillating component. Since the VLBI observations were carried out in dual-polarization mode, we will also have a closer look to the polarization analysis. In addition, we will work out the spectral properties of the individual jet components using the three available frequencies. We further plan to continue the single dish monitoring in order to find out if and when $0917+624$ is going to return to its former prominent IDV activity.

\section{Acknowledgments}

We thank the MOJAVE and $2 \mathrm{~cm}$ Survey programs for providing their data. We appreciate the 
use of the flux density monitoring data from the UMRAO data base. This work has made use of the VLBA, which is an instrument of the National Radio Astronomy Observatory, a facility of the National Science Foundation, operated under cooperative agreement by Associated Universities, Inc. This work is also based on observations with the 100-m radio telescope of the MPIfR (MaxPlanck-Institut für Radioastronomie) at Effelsberg.

\section{References}

[1] A. Quirrenbach et al., Statistics of intraday variability in extragalactic radio sources, A\&A (1992), vol. 258, no. 2, p. 279-284.

[2] S. Wagner \& A. Witzel, Intraday Variability In Quasars and BL Lac Objects, ARA\&A (1995), vol. 33, pp. 163-198.

[3] L.L. Kedziora-Chudczer et al., The ATCA intraday variability survey of extragalactic radio sources, MNRAS (2001), vol. 325, Issue 4, pp. 1411-1430.

[4] K.E. Gabanyi et al., Effects of the turbulent ISM on radio observations of quasars, in proceedings of 8th EVN Symposium, .

[5] A. Quirrenbach et al., Rapid radio polarization variability in the quasar $0917+624, A \& A$ (1989), vol. 226, no. 1, p. L1-L4.

[6] A. Kraus et al., Intraday variability in compact extragalactic radio sources. II. Observations with the Effelsberg 100 m radio telescope, A\&A (2003), vol. 401, p. 161-172.

[7] K.J. Standke et al., The intraday variable quasar 0917+624: VLBI and X-ray observations, A\&A (1996), vol. 306, p. 27.

[8] A. Kraus et al., A change in the variability properties of the intraday variable quasar $0917+624, A \& A$ (1999), vol. 352, p. L107-L110.

[9] L. Fuhrmann, Investigations of Intraday Variable Blazar Cores and the Connected Interstellar Medium, PhD Thesis (2004), University of Bonn

[10] L. Fuhrmann et al. Annual Modulation in the Variability Properties of the IDV Source 0917+624?, PASA (2002), vol. 19, Issue 1, pp. 64-68.

[11] U. Bach et al., Kinematic study of the blazar S5 0716+714, A\&A (2005), vol. 433, Issue 3, pp. 815-825.

[12] N. Kudryavtseva, The kinematics of 1803+784, in proceedings of 8th EVN Symposium, .

[13] T.P. Krichbaum et al., Intraday Variability in Northern Hemisphere Radio Sources, PASA (2002), vol. 19, Issue 1, pp. 14-18.

[14] M. L. Lister and D.C. Homan, MOJAVE: Monitoring of Jets in Active Galactic Nuclei with VLBA Experiments. I. First-Epoch 15 GHz Linear Polarization Images, AJ (2005), vol. 130, 1389.

[15] K. I. Kellermann et al., Sub-Milliarcsecond Imaging of Quasars and Active Galactic Nuclei. III. Kinematics of Parsec-scale Radio Jets, ApJ 2004, vol. 609, Issue 2, pp. 539-563.

[16] B. Rickett et al. Interstellar scintillation of the radio source 0917+624, A\&A (1995), vol. 293, 479-492.

[17] J.L. Gomez et al. Hydrodynamical Models of Superluminal Sources, ApJL (1997), vol. 482, p. L33. 